\title{
Dynamic Channel Allocation in IEEE 802.11 Networks
}

\author{
Somenath Chakraborty \\ $\mathrm{PhD}$ Research Scholar \\ Computer Science \& Engineer- \\ ing \\ Calcutta University
}

\author{
Banani Saha, PhD \\ Associate Professor \\ Computer Science \& Engineer- \\ ing \\ Calcutta University
}

\author{
Samir K. Bandyopadhyay \\ Professor \\ Computer Science \& Engineer- \\ ing \\ Calcutta University
}

\begin{abstract}
Wireless channel allocation plays as an important role in thedesign of wireless network, as it greatly influences the throughput and performance of the network. It is proposed a technique to improve the usage of wireless spectrum in the context of IEEE 802.11 wireless network using new channel assignment methods among interfering Access Points (APs). Reliable channel selection for each Access Point (AP) is essential in setting up and operating densely deployed 802.11 WLANs. The aim of the channel selection is to provide efficient reuse of the spectrum and therefore minimize interference and improve the performance. The focus is mainly on to design an algorithm to solve the problem of channel allocation using Simulated Annealing. The algorithm minimizes the interference among the Access Points (APs). All the access points in the network operate on this algorithm simultaneously and determine the best channel it should use to minimize the interference from the neighboring access points.
\end{abstract}

\section{Keywords}

Wireless Network, Network Interference, Dynamic channel allocation, Co-channel interference factor, Access Point Optimization

\section{INTRODUCTION}

A key issue in Wireless LANs (WLANs) is the management of user congestion through proper channel allocation which is very limited. Interference among the channels degrades the performance of the network and it is a very challenging issue as the network density is increasing exponentially. This paper proposed a heuristic approach for dynamic channel allocation in wireless LAN. The heuristic approach mainly focuses on the optimization of the performance by decreasing the channel interference by the dynamically choose the most appropriate channel on that particular instance and the instance changes as because the environment changes with the change of the user's need.

\section{HISTORICAL SURVEY}

Many research articles have been published regarding allocation of channel. With the exponential growth of wireless uses it is still a challenging research field for allocation of channel in a dynamic environment which optimizes the channel interference. Among them were those by Al Mamun et al.[1], This paper proposes a variable channel allocation technique based on the bandwidth requirement and Quality of Service (QoS) of the network. But this work lacks time complexity issues as it depends on Quality of Service (QoS).

Chen et al.[2] introduce a distributed version of simulated annealing to solve the dynamic channel allocation problem in high-density WLANs. The approach is generic and applicable to any access system where channels are not allocated in a prefixed or centralized manner. This algorithm is scales well and the in different networks it provides good results but the problem is that it cannot provide near actual results and sometimes it cannot able to produce good approximation values for the channel allocations. Robert Akl et al.[4] provides a method for dynamic channel allocation in two different way. One they choose as a random pick-up of channel where the performance dwindles with the increase of APs in a certain instance of time .Another way they choose as first pick-up of channels where the nearest and strongest one is selection where also issues arises for performance decreasing due to overlapping of channels and interference. Y. Lee et al. [5] proposes the method of channel allocation depending upon the frequency distribution where the issues arises like signal strength mismatch and nearest AP selection problems. Frequency distribution becomes very challenging where the situation arises like a set of channels having near equal frequencies, in that case interference is the maximum observed. Surachai Chieochan et al,[6] present an survey on existing research works[7-16] on channel allocation in IEEE 802.11 based wireless networks. They also give a comparison showing the execution behavior, complicity and scalability of the recent development algorithms on channel allocation based on IEEE 802.11.

This paper proposes a novel dynamic approach of channel allocation depending on the environmental situation to minimizing the interference effects in all possible ways, hence improving the performance of channel allocation in 802.11 wireless networks.

\section{PROPOSED METHOD}

Here we briefly describe the procedure that we proposed for optimization in Wireless LAN on a dynamic basis. As the network is synchronized, so the algorithm works as per the rule and provides the optimize results in the dynamic channel allocation problem. We frame the problem as a optimization problem and solve it accordingly.

\subsection{Input parameters}

1. $\mathrm{A}_{\mathrm{i}}$ is the set of neighboring $\mathrm{APs}$ to $\mathrm{AP}_{\mathrm{i}}$.

2. $\mathrm{d}_{\mathrm{ij}}$ is the distance between $\mathrm{AP}_{\mathrm{i}}$ and $\mathrm{AP}_{\mathrm{j}}$.

3. $\mathrm{Fi}$ is the channel assigned to APi .

4. Iij is the interference that APj causes on APi.

5. Iij is the interference that $\mathrm{AP} \mathrm{j}$ causes on $\mathrm{AP} \mathrm{i}$.

6. $\mathrm{K}$ is the total number of available channels.802.11 $\mathrm{b} / \mathrm{g}$ has 11 channels.

7. Path Loss is a function that captures the attenuation loss based on the COST Hata model.

8. $\mathrm{P}_{\mathrm{i}}$ is the transmit power of $\mathrm{AP} \mathrm{i}$.

9. $\mathrm{Q}_{\mathrm{i}}$ is the cardinality of $\mathrm{A}_{\mathrm{i}}$. 
10. OIFij is the overlapping channel interference factor between $\mathrm{AP} i$ and $\mathrm{AP} j$.

\subsection{Visualization using MATLAB:}

We implement our optimization problem in MATLAB so that we can visualize the data as well as can decide the best possible way to choose the used parameter values.

\subsection{Overlapping Interference values for two cases of implementation}

\subsection{Objective Function}

The objective function for dynamic channel allocation problem

is given as :----

$$
\underset{\mathrm{F}_{\mathrm{i}}}{\operatorname{Min}} \sum_{j=1}^{Q i} \mathbf{I} \mathrm{ij},
$$

Here we show two references of the output, it can be easily decide that the second values contains mostly zeros which itself infer the optimization success of our algorithm. It is showing that it greatly optimizes the optimization problem and hence having these values.

Subject to $\mathrm{I}_{\mathrm{ij}}=\frac{(\text { OIF } i j) * P}{\text { Pat } h \text { Loss }}$ for $\mathrm{j} € \mathrm{~A}_{\mathrm{i}}$ and $\mathrm{F}_{\mathrm{i}} €\{1, \ldots, \mathrm{K}\}---(1)$ Constraint (1) defines the interference between $\mathrm{AP}_{\mathrm{i}}$ and $\mathrm{AP}_{\mathrm{j}}$.

\subsection{Algorithm}

During every $\mathrm{T}$ second time-interval:

1. Report traffic and Received Signal strength (RSS) to server.

2. Compute co-channel interference between any two cells using the constraint (1) given.

3. For minimizing the constraint (1), Simulated annealing is used by which choose the assignment in such a way by which the interference between AP i and $\mathrm{AP} \mathrm{j}$ should be minimum.

4. APs change channels. Broadcast change to stations within 1 to $2 \mathrm{~ms}$.

\section{EXPERIMENTAL RESULTS}

This section provides indicative evidence on the performance of our proposed algorithms, by applying them to a set of experimental data. MATLAB 7.0.4.365(R14) Service pack 2 is used for the coding and visualization purpose. We define $11 \times 14$ grid of Acces Point forming a WLAN. Path Loss model calculate the path loss according to the Table 1 shown below.

\begin{tabular}{|l|l|l|}
\hline Parameter & Name & Value \\
\hline $\mathrm{R}$ & Radius of a cell & 0.5 to $5 \mathrm{Km}$ \\
\hline $\mathrm{P}$ & $\begin{array}{l}\text { The transmit power of individual } \\
\text { AP }\end{array}$ & $20 \mathrm{dBm}$ \\
\hline hte & $\begin{array}{l}\text { Effective transmitter antenna } \\
\text { height }\end{array}$ & 30 to $200 \mathrm{~m}$ \\
\hline hre & Effective receiver antenna height & 1 to $10 \mathrm{~m}$ \\
\hline fc & Frequency of the carrier wave & 150 to $1500 \mathrm{MHz}$ \\
\hline
\end{tabular}
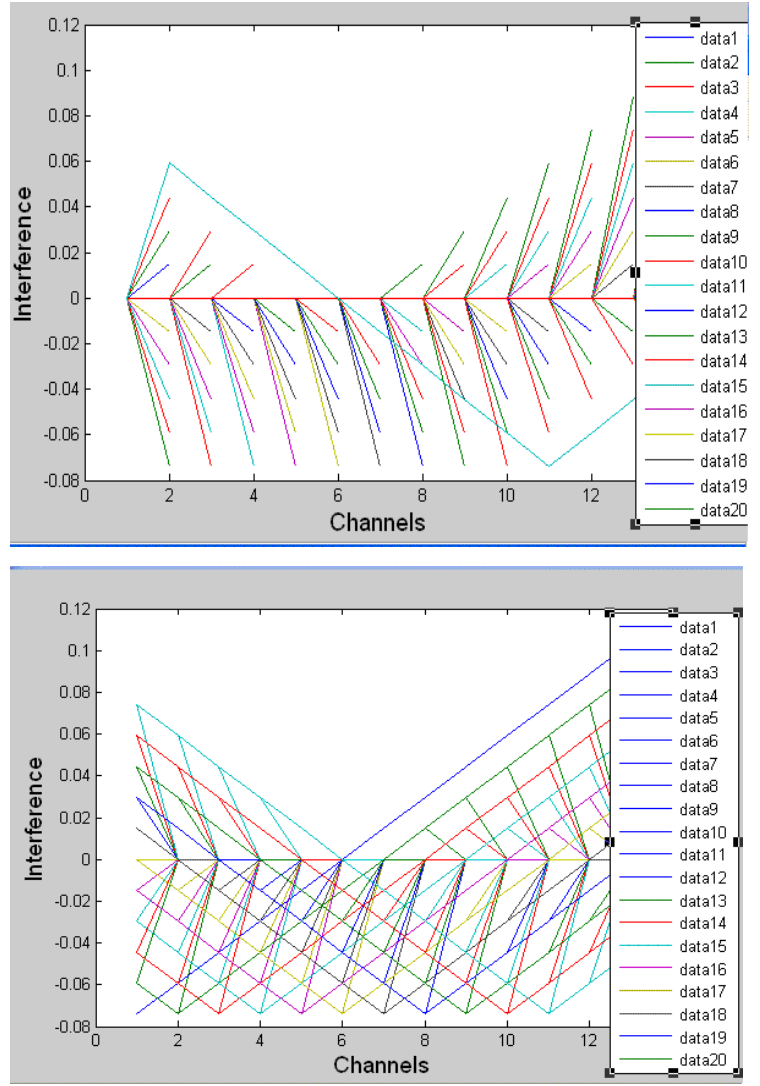

Fig 1: a) without the proposed method b) with the use of

\begin{tabular}{|c|c|c|c|c|c|c|c|c|c|c|}
\hline 1 & ? & & & & & & & & & \\
\hline 0.010707 & .019529 & .00444 & 002633 & 0014915 & & 0.01486 & 00063 & 004444 & 0.68969 & 0.0740. \\
\hline .0.1652599 & .007074 & .068569 & .004444 & 000263 & .00191515 & & 0001815 & 0.02020 & 0.04444 & 0.0592 \\
\hline .004444 & 0.06929 & .007474 & $\cdot 0.65959$ & 0.0444 & .020263 & .0 .014815 & & 0014915 & 00263 & 10044 \\
\hline 002633 & 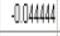 & 0.069269 & .0074014 & .056929 & volu44 & 0.0263 & 0.014815 & & 0.0419615 & 0008 \\
\hline .00148615 & 0.0833 & .0.4444 & -0.65959 & 0.014707 & .0 .06969 & 0.04444 & .00263 & .00191515 & & 00,1481 \\
\hline & .001815 & 0.02603 & 0.04444 & ALESESO & .007474 & .0 .65259 & 0.04444 & 0.02830 & .0014815 & \\
\hline 00148915 & & .0014065 & 002683 & v0.444.4 & 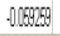 & .0104014 & 0.05959 & .00444 & 0.02833 & 0.01696 \\
\hline 002633 & 0001915 & & -0.014815 & $-1020 \% 3$ & N044444 & .0 .163299 & 0074074 & . 1099259 & .0 .0444 & 10028 \\
\hline 0,04444 & 000863 & 00019915 & & 0.01415 & 0.0263 & .0 .04444 & .0.05959 & .0707074 & .0 .165699 & 0.0444 \\
\hline 0.65959 & 0.0444 & 0,0263 & 0014815 & & .001915 & 0.0283 & 0.04444 & .016969 & .007474 & 0.09525 \\
\hline 0.074074 & 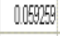 & 0.04444 & 000263 & 00014915 & & .00148615 & 6.00833 & volutul & .0 .16969 & 100747 \\
\hline 0.080009 & 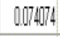 & {$[16929$} & 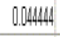 & 0.0283 & 00191915 & & 0.014619 & 0.02603 & Nontul & 0.09925 \\
\hline 0.1067 & 00000009 & 0.074714 & 0.065999 & 004444 & 0.02503 & 0.04616 & & .001961 & (1020263 & 0.0444 \\
\hline 0.11182 & $01037]$ & & & & 0.044 & & & & 0.014619 & .009 \\
\hline
\end{tabular}
proposed method.

Result values without optimization method used

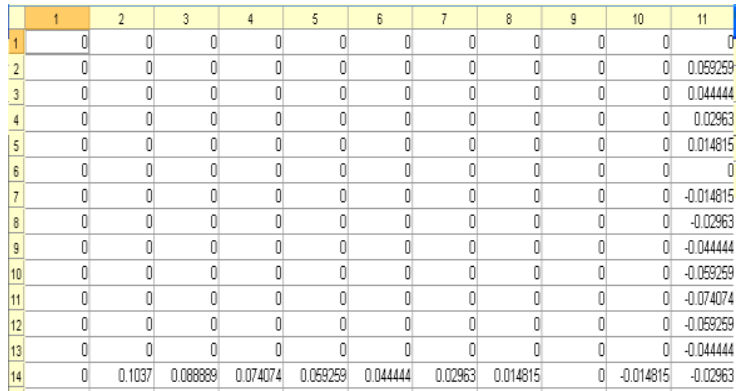

Here the optimized values are shown using our proposed method. 


\section{CONCLUSION}

Our dynamic channel allocation problem greatly reduced the overlapping channel interference. It can apply in any distributed IEEE 802.11 WLAN and most importantly it is working on dynamic environments and all the set-up changes rapidly with the changing environments hence reducing frequency overlapping issues and stacking of channel uses and channel barrier issues. But with the exponential growth of the wireless technology there is a huge scope left for the future developments of our project as the demands arises regarding access time minimization and quick response in real time environments. As the multimedia data transform is a key issues of wireless technology at the present time we need to develop the system which can perform in real time mode and optimize the throughput in all respects.

\section{REFERENCES}

[1] Al Mamun, K.M.A. et.al, "An Efficient Variable Channel Allocation Technique for WLAN IEEE 802.11 Standard", in Proceedings Conference on Circuits, Communication and System, pp.92-95.2009.

[2] Chen, J. et.al, "A Fast Channel Allocation Scheme Using Simulated Annealing in Scalable WLANs", IEEE International Conference on Broadband Communications, Networks and System, pp.205-211. 2008.

[3] V. Cerny, "A thermodynamically approach to the traveling salesman Problem: an efficient simulation algorithm,"Journal of Optimization Theory and Applications, vol. 45, pp.41-51, 1985 .

[4] Robert Akl, Anurag Arepally, "Dynamic Channel Assignment in IEEE 802.11 Networks", IEEE International Conference on Portable Information Devices, 2007. PORTABLE07.

[5] Y. Lee, K. Kim, and Y. Choi, "Optimization of AP placement and channel Assignment in wireless LANs", LCN 2002. 27th Annual IEEE Conference on Local Computer Networks, pp. 831-836, November 2002.

[6] Surachai Chieochan, Ekram Hossain, and Jeffrey Diamond, "Channel Assignment Schemes for Infrastructure-Based 802.11 WLANs: A Survey", IEEE COMMUNICATIONS SURVEYS \& TUTORIALS, VOL. 12, NO. 1, FIRST QUARTER 2010.

[7] A.Mishra and V. Shrivastava, D. Agrawal, S. Banerjee, and S. Ganguly, "Distributed Channel Management in Uncoordinated Wireless Environments," in Proc. International Conference on Mobile Computing and Networking, pp. 170-181, 2006.

[8] Y. Bejerano, S. Han, and L. Li, "Fairness and Load Balancing in Wireless LANs Using Association Control," in Proc. ACM Mobicom, 2004.

[9] A. Akella, G. Judd, S. Seshan, and P. Steenkiste, "SelfManagement in Chaotic Wireless Deployments," in Proc. ACM Mobicom, 2005.
[10] A. Vasan, R. Ramjee, and T. Woo, "Echos: Enhanced Capacity 802.11 Hotspots," in Proc. IEEE Infocom, 2005.

[11] J. K. Chen, G. D. Veciana, and T. S. Rappaport, "Improved Measurement-based Frequency Allocation Algorithms for Wireless Networks,"in Proc. IEEE GLOBECOM'07, Washington, DC, USA, Nov.2007 .

[12] M. Haidar, R. Akl, and H. Al-Rizzo, "Channel Assignment and Load Distribution in a Power-managed WLAN," in Proc. IEEE PIMRC: 18th Annual IEEE International Symposium on Personal, Indoor and Mobile Radio Communications, September 2007.

[13] H. Al-Rizzo, M. Haidar, R. Akl, and Y. Chan, "Enhanced Channel Assignment and Load Distribution in IEEE 802.11 WLANs, in Proc. IEEE International Conference on Signal Processing and Communication, November 2007

[14] Handrizal, Noraziah Ahmad,Ahmad N Abda Alla," Comparison Between Direct Sequence Spread Spectrum (DSSS) And Frequency Hopping Spread Spectrum (FHSS)", in proceedings of ICSECS'09, vol. 1, October 2009.

[15] A. Mishra, S. Banerjee, and W. Arbaugh, "Weighted coloring based Channel assignment for WLANs," ACM SIGMOBILE Mobile Computing and Communications Review, vol. 9, pp. 19-31, 2005.

[16] Tekinay, S. and Jabbari, B. 'Handover and channel assignment in mobile cellular networks',IEEE Communications Magazine, Vol. 29, No.11, pp.42-46,1991.

[17] W. K. Hale, "Frequency Assignment: Theory and Applications", in Proc. IEEE, Vol. 68, pp. 1497-1514, 1980.

[18] "Using Radio Resource Management to Deliver Secure and Reliable WLAN Services," http://www.cisco.com/en/US/prod/collateral/wireless/ps6 302/ps8322/ps6307/prod white paper0900aecd802c949b ns736 Networking Solutions White Paper.html, Available Online.

[19] "IEEE Standard for Information TechnologyTelecommunications and Information Exchange between Systems-Local and Metropolitan Area Networks-specific Requirements - Part 11: Wireless LAN MediumAccess Control (MAC) and Physical Layer (PHY) Specifications," IEEE STD 802.11-2007 (Revision of IEEE Std 802.11-1999), pp. C1-1184, June 122007.

[20] "Supplement to IEEE Standard for Information Technology Telecommunications and Information Exchange between Systems - Local and Metropolitan Area Networks - Specific Requirements. Part 11: Wireless LAN Medium Access Control (MAC) and Physical Layer (PHY) Specifications: High-Speed Physical Layer in the 5 GHz Band,” IEEE STD 802.11a-1999, 1999.

[21] "List of WLANs Channels," http://en.wikipedia.org/wiki/List of WLAN channels, Available Online. 